\title{
Spectral characteristics of PMMA doped with a dimethacrylate derivative of naphthalene-2,7-diol use full in UV sensors
}

\author{
Malgorzata Gil ${ }^{1}$, Beata Podkościelna ${ }^{2}$, Lidia Czyzewska ${ }^{1}$, Janusz Pędzisz ${ }^{1}$, Aleksander Walewski ${ }^{1}$, Jaroslaw Kopec ${ }^{1}$, \\ Wieslaw Podkoscielny ${ }^{1}$, Andrzej Gorgol ${ }^{1}$, Pawel Mergo ${ }^{1}$ \\ ${ }^{1}$ Laboratory of Optical Fibre Technology, Maria Curie-Sklodowska University, Pl. Sklodowskiej 3, 20-031 Lublin, \\ Poland \\ ${ }^{2}$ Departament of Polymer Chemistry, Maria Curie-Sklodowska University, Gliniana St. 33, 20-031 Lublin, \\ Poland
}

Received December 28, 2015; accepted March 29, 2016; published March 31, 2016

\begin{abstract}
The paper presents a comparison of spectral characteristics of PMMA samples doped with a new photoluminescence monomer dimethacrylate derivative of naphthalene-2,7-diol (2,7NAF.DM). After excitation by UV radiation the monomer emits yellowgreen light. One PMMA sample was doped in a bulk thermal polymerization process. The second was doped by diffusion from a methanol solution. A shift of the emission spectrum was observed in the sample doped in bulk thermal polymerization. Both PMMA samples may be used in polymer UV sensors.
\end{abstract}

Due to specific material properties, the technology of polymer optical fibers (POF) has been intensively developed in recent years. Polymer fibers may show better parameters than fibers made of silica glass in a variety of applications. One of the advantages is biological compatibility, which opens the possibility for medical applications. Moreover, polymers have higher flexibility than silica glass, therefore can withstand much higher strains, which enables applications of polymer fibers as elongation sensors in a strain range unattainable for silica fibers [1]. Also polymers offer a relatively easy possibility to incorporate inorganic and organic dopants after material polymerization and/or fiber drawing to alter their optical properties.

Photoluminescent monomers and polymers are contemporary and leading materials, which find applications in the rapidly developing areas of optoelectronics or photonics [2-8]. These unique materials may be used, e.g. as organic projectors and in construction of energy saving light sources [9]. Therefore, demand for new and more resistant photoluminescence materials is still increasing, becoming a challenge for researchers. Inorganic materials so far used in photoluminescence, e.g. zinc sulfide with copper and cobalt admixture or inorganic aluminum compounds are changed for reactive and more accessible organic compounds [10-13]. These compounds may be introduced into a polymer matrix in the bulk material polymerization process or by the diffusion process from the solution.
In this letter we present the spectral characteristics of polymethylmetacrylate (PMMA) doped with a new photoluminescence monomer dimethacrylate derivative of naphthalene-2,7-diol (2,7-NAF.DM). We present a comparison of two samples of PMMA. One was doped in bulk polymerization process. The second was doped by diffusion from a methanol solution.

The new photoluminescent monomer 2,7-NAF.DM was synthesized in the Department of Polymer Chemistry UMCS in the two-step reaction. In the first step, naphthalene-2,7-diol (2,7.NAF) was reacted with epichlorohydrin. Next, esterification of the obtained diglycidyl ether was carried out with the use of methacrylic acid in the presence of hydroquinone (polymerization inhibitor) and triethylbenzylammonium chloride as a catalyst. The detailed information about synthesis was presented in $[14,15]$.

The absorption (Fig. 1), excitation (Fig. 2) and emission (Fig. 3) spectra for the 2,7-NAF.DM were measured.

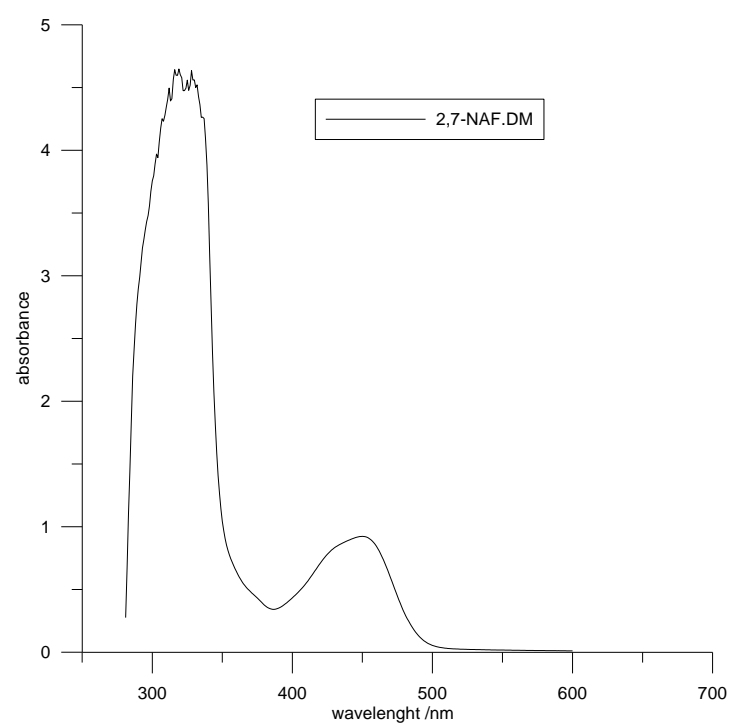

Fig. 1. The absorption spectrum of 2,7-NAF.DM. 
Excitation maximum for 2,7-NAF.DM is located near $550 \mathrm{~nm}$. After excitation by UV radiation, the sample emits yellow-green light, which coincides with the range of a wavelength for the green light (500-542nm) and for the yellow one $(542-578 \mathrm{~nm})$.

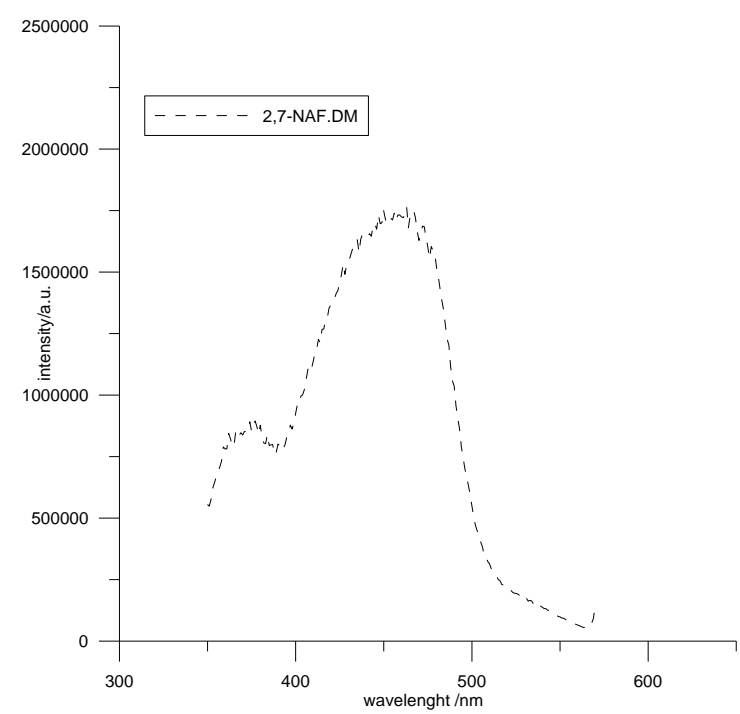

Fig. 2. Excitation spectrum of 2,7-NAF.DM

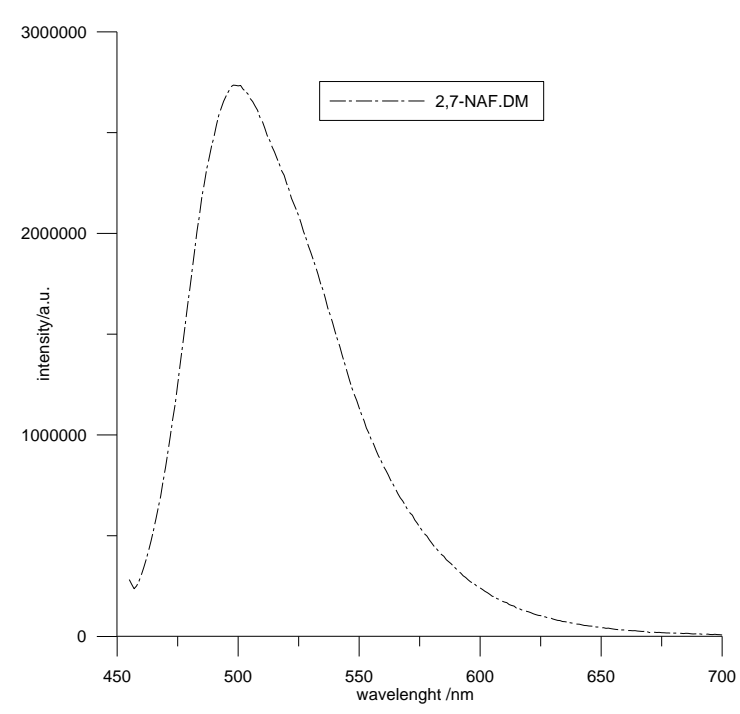

Fig. 3. Emission spectrum of 2,7-NAF.DM.

On the basis of our previous works [16] the doped PMMA was fabricated by thermal polymerization of the mixture: methylmetacrytate (MMA) as a basic monomer, dimethacrylate derivative of naphthalate-2,7-diol as a dopant $(5 \% \mathrm{w} / \mathrm{w})$, benzoyl peroxide as an initiator $(0.4 \%$ $\mathrm{w} / \mathrm{w})$ and thioglycolic acid as a chain transfer agent $(0.8 \%$ $\mathrm{w} / \mathrm{w})$.

http://www.photonics.pl/PLP
For diffusion doping, a pure PMMA was fabricated by thermal polymerization of the mixture: methylmetacrytate (MMA), benzoyl peroxide $(0.4 \% \mathrm{w} / \mathrm{w})$ and thioglycolic acid $(0.8 \% \mathrm{w} / \mathrm{w})$.

Both fabricated PMMA samples had a cylindrical shape with a diameter of $15 \mathrm{~mm}$ and a length of $200 \mathrm{~mm}$. From each sample a flat plate about $20 \mathrm{~mm}$ thick was cut.

Diffusion doping was performed by immersing the pure PMMA plate in a methanol solution of 2,7-NAF.DM (1.1 $\%$ w/w). The diffusion process took 2 hours. Next, the plate was dried in a flow of dry inert gas (argon) at room temperature for 2 days and then at $70^{\circ} \mathrm{C}$ for 1 day, which allowed a complete removal of methanol.

For fabricated doped polymers, spectral characteristics were made. The measurements were performed by using: supercontinuum SuperK Extreme (NKT Photonics) with a short wave UV filter as a light source and an optical spectrum analyzer ANDO AQ 6315E. The light generated by the source (SC) and then filtered through a short wave UV filter was introduced into the multimode fiber. The end of this fiber illuminated the doped polymer plates. The generated photoluminescent light was collected by a second multimode optical fiber placed on the sample side and analyzed by the optical spectrum analyzer. Figure 4 presents the spectrum of generated light by the sample doped before PMMA polymerization. Figure 5 presents the spectrum of generated light by the sample doped by diffusion.

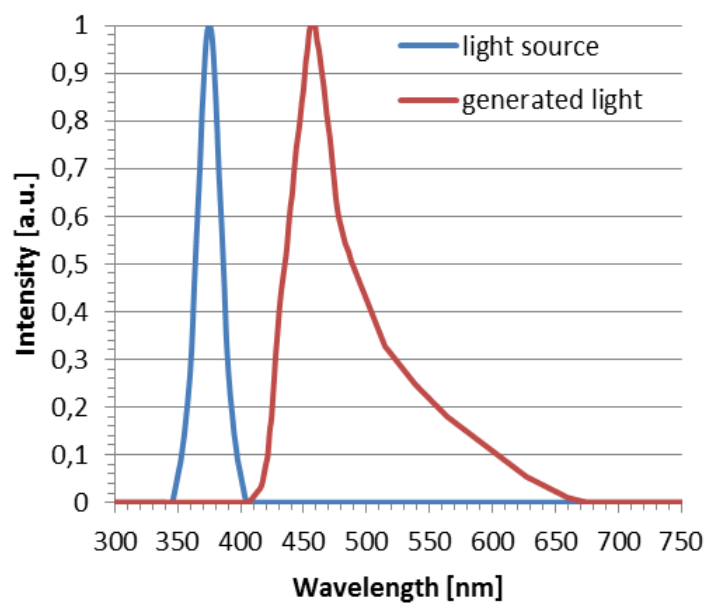

Fig. 4. Spectrum of light source and generated light for PMMA sample doped before polymerization.

On the basis of measurements, it can be seen that the photoluminescence maximum for the PMMA doped before polymerization is shifted approximately $40 \mathrm{~nm}$ in the direction of a shorter wavelength compared to the emission spectrum of a 2,7-NAF.DM monomer (Fig. 3). Such a shift is not observed for PMMA doped by 
diffusion. This is probably due to the mutual interaction of MMA and 2,7-NAF.DM monomers during polymerization. The fabricated copolymer is probably crosslinked. Trapped in the PMMA network, the molecule of a 2,7-NAF.DM monomer loses, after excitation, some energy, which results in a shift of the maximum of the emission spectrum.

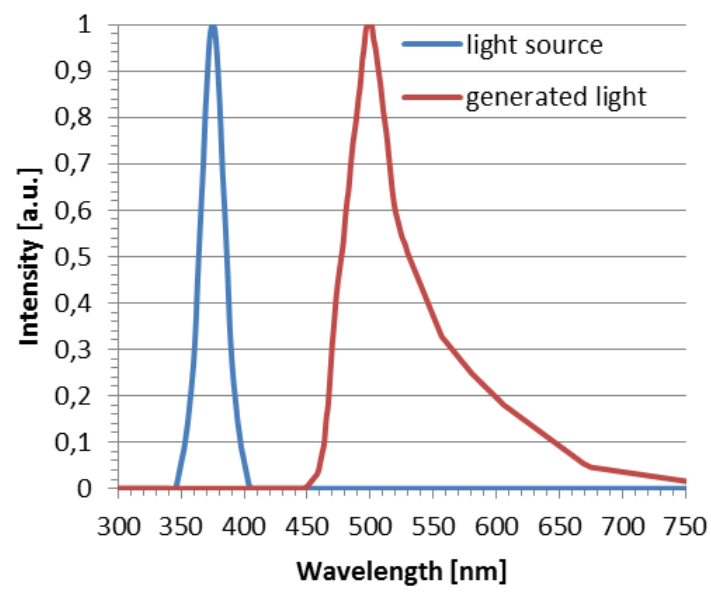

Fig. 5. Spectrum of a light source and generated light for the PMMA sample doped by diffusion.

For both samples the power of emitted light was measured. For these measurements, new cylindrical samples were prepared (with the same procedure as presented above). Each of them had a diameter of $7 \mathrm{~mm}$ and a length of $20 \mathrm{~mm}$. Measurements of illuminated and emitted light were made with a Thorlabs power meter PM100 with a measuring head S120UV. The power of emitted light was measured by the direct end of the samples close to the measuring head. For both samples the power of illuminated light was equal to $0.05 \mathrm{~W}$. The power of emitted light for the sample doped before PMMA polymerization was equal to $0.047 \mathrm{~W}$. The calculated luminescence efficiency is equal to 0.94 . For the sample doped by diffusion the power of emitted light was equal to $0.028 \mathrm{~W}$. A much lower value arises from a significantly smaller quantity of dopant introduced into the samples. The calculated luminescence efficiency is equal to 0.56 .

In this study, a comparison of two doped PMMA samples was presented. Both samples were doped with a new 2,7NAF.DM monomer. The generation of green-yellow light was observed after each samples excitation with UV light. Thanks to this, the presented doped polymers may be used in the construction of UV sensors
The research leading to these results has received funding from the People Programme (Marie Curie Actions) of the European Union's Seventh Framework Programme FP7/2007-2013/ under REA grant agreement no 608382 .

\section{References}

[1] D. Webb, K. Kalli, "Polymer fiber Bragg gratings" in Fiber Bragg Grating Sensors: Recent Advancements, Industrial Applications and Market Exploitation, eds.: A. Cusano, A. Cutolo, J. Albert, (Bentham Science Publishers 2011), pp. 292-312.

[2] D.Y. Kim, H.N. Cho, C.Y. Kim, Prog. Polym. Sci. 11, 4450 (2000).

[3] J.M. Hong, H.N. Cho, D.Y. Kim, C.Y. Kim, Synthetic Met. 102, 933 (1999).

[4] D. Vollath, D.V. Szabo, S. Schlabach, J. Nanopart. Res. 6, 181 (2004).

[5] S.H. Chen, M. Ando, N. Murase, Materials Lett. 65, 3146 (2011).

[6] J. Tang, Y. Wang, D. Yu, Z. Zhou, C. Wang, B. Yang, Materials Lett. 50, 371 (2001).

[7] C. Zhang, J. Lin, Chem. Soc. Rev. 41(23), 7938 (2012).

[8] L. Museur, P. Gorbovyi, M. Traore,A. Kanaev, L. Rozes, C. Sanchez, J. Lumin. 132, 1192 (2012).

[9] Y. Hu, Y. Zhang, J. Gao, Adv. Mater. 18, 2880 (2006).

[10] T. Mori, M. Kijima, Eur. Polym. J. 45, 1149 (2009).

[11] M. Montalti, L.S. Dolci, L. Prodi, N. Zaccheroni, M.C.A. Stuart, K.J.C. van Bommel, Langmuir 22, 2299 (2006).

[12] M. Tomasulo, S.L. Kaanumal, S. Sortino, F.M. Raymo, J. Org. Chem. 72, 595 (2007).

[13] F. Huang, Y. Zhang, M.S. Liu, Y.J. Cheng, A.K.Y. Jen, Adv. Funct. Mater. 17, 3808 (2007).

[14] B. Podkościelna, B. Gawdzik, Appl. Surf. Sci. 256, 2462 (2010).

[15] B. Podkościelna, J. Therm. Anal. Calorim. 116, 785 (2014).

[16] P. Mergo, M. Gil, K. Skorupski, J. Klimek, G. Wójcik, J. Pędzisz, J. Kopec, K. Poturaj, L. Czyzewska, A. Walewski, A. Gorgol, Phot. Lett. Pol. 5, 170 (2013). 